\title{
Multiple observations of quantum clocks
}

\author{
V. Bužek, ${ }^{1, *}$ P. L. Knight, ${ }^{2}$ and N. Imoto ${ }^{1}$ \\ ${ }^{1}$ CREST Research Team for Interacting Carrier Electronics, School of Advanced Sciences, \\ The Graduate University for Advanced Studies (SOKEN), Hayama, Kanagawa, 240-0193, Japan \\ ${ }^{2}$ Optics Section, The Blackett Laboratory, Imperial College, London SW7 2BW, United Kingdom
}

(Received 9 May 2000; published 10 November 2000)

\begin{abstract}
How much information about the original state preparation can be extracted from a quantum system which has already been measured? That is, how many independent (noncommunicating) observers can measure the quantum system sequentially and give a nontrivial estimation of the original unknown state? We investigate these questions, and show from a simple example that information about the original preparation is not entirely lost as a result of the measurement-induced collapse of the quantum state, and that an infinite number of independent observers who have no prior knowledge about the initial state can gain partial information about the original preparation of the quantum system.
\end{abstract}

PACS number(s): 03.65.Bz, 42.50.Ar, 89.70.+c

From the deterministic measurement model employed in classical physics, it follows that the state of the physical system is not affected by measurement. That is, information about states of the system can be determined with an arbitrary precision. Formally, from a kinematical point of view, this can be expressed as follows: in classical physics there are measurements $(m)$ for which the statistics of the measurement results $(r)$ characterized by the conditional probability distribution $p_{m}(r \mid s)$ can be, for all possible states $s$ of the given classical system, of the form

$$
p_{m}(r \mid s)=\delta\left(s_{r}-s\right) .
$$

Moreover, these measurements do not change the state of the classical system, so an arbitrary number of independent observers (i.e., observers who do not communicate) can determine the state.

The standard Copenhagen interpretation of quantum mechanics is deeply rooted in a model of nondeterministic statistical measurement [1]. From the kinematical point of view, the quantum theory models (predicts) the statistics of results registered by a measuring device when the measurement is performed on a quantum object. Within this nondeterministic model of measurement, the conditional probability distribution $p_{m}(r \mid s)$ can never be of the form of Eq. (1) for arbitrary, initially unknown, states of a quantum system. In quantum mechanics the conditional probability distribution $p_{m}(r \mid s)$ is given by the expression

$$
p_{m}(r \mid s)=\operatorname{Tr}\left[\hat{O}_{r} \hat{\rho}_{s}\right]
$$

where the set of positive operators $\hat{O}_{r}$ which sum up to the identity operator models the measuring device, and the den-

\footnotetext{
*Permanent address: Institute of Physics, Slovak Academy of Sciences, Dúbravská cesta 9, 84228 Bratislava, Slovakia. Faculty of Informatics, Masaryk University, Botanická 68a, 60200 Brno, Czech Republic.
}

sity matrix characterizes the state of the quantum object that is the subject of the measurement.

The axiomatics of quantum theory implicitly require that the state of the system is changed during measurement. Otherwise, repeated measurements of the previously measured but unchanged quantum state could reveal still more information about the state. Consequently, the measurement model would eventually be equivalent to the standard deterministic measurement model of classical physics. Therefore, there is an additional rule which excludes the possibility of repeated measurements. This additional principle is the wellknown von Neumann projection postulate.

Nevertheless it is an interesting question to ask how much information about the original state is "left" in a system which has already been measured. That is, how much information about the preparation can be extracted from the system by a second observer who does not communicate with the first observer. A further question we would like to understand is whether, from the axioms of quantum theory, we can obtain a "classical-like" picture when a physical system in an unknown state can be repeatedly measured, yet still retain information about the original state preparation. In what follows we analyze a simple example which illuminates these two questions.

First we specify the task of measurement. In measurement we wish to determine some parameters of the state of a quantum system which correspond to a symmetry group. As an example, consider a position measurement which is connected with a group of translations, or a measurement of the angle of orientation connected with a group of rotations. In what follows we analyze the simplest example of a continuous parameter $\varphi \in\langle 0,2 \pi\rangle$, the phase which parametrizes the group of rotations in the two-dimensional space of the $U(1)$ group. To make our discussion more physical we consider a model of optimal quantum clocks discussed in our previous work [2]. We will analyze the situation when the observers have no a priori knowledge about the original state preparation. This means that the prior phase distribution is constant and equal to $1 / 2 \pi$. 
In our previous paper we studied the problem of building an optimal quantum clock from an ensemble of $N$ ions [3]. In particular we assumed an ion trap with $N$ two-level ions, all in the ground state $|\Psi\rangle=|0\rangle \otimes \cdots \otimes|0\rangle$. This state is an eigenstate of the free Hamiltonian, and thus cannot record time (phase). Therefore, the first step in building a clock was to bring the system to an appropriate initial (reference) state $\hat{\Omega}$ which is not an energy eigenstate. For instance, one can apply a Ramsey pulse whose shape and duration is chosen such that it puts all the ions into the product state

$$
\hat{\Omega}=\hat{\rho}^{\otimes N},
$$

with $\hat{\rho}=|\psi\rangle\langle\psi|$ and $|\psi\rangle=(|0\rangle+|1\rangle) / \sqrt{2}$. After this preparation stage, the ions evolve in time according to the Hamiltonian evolution $\hat{\Omega}(t)=\hat{U}(t) \hat{\Omega} \hat{U}^{\dagger}(t)$, where $\hat{U}(t)$ $=\exp \{-i t \hat{H}\}$ (we use units such that $\hbar=1$ ). Therefore, these ions can be viewed as a time-recording device. The task is to determine this time $t$ (or equivalently the corresponding phase) by carrying out a measurement on the ions. Note that because of the indeterminism of quantum mechanics it is impossible, given a single set of $N$ two-level ions, to determine the elapsed time with certainty. As we showed earlier [4], one can find an optimal measurement (see below), with the help of which information about the phase can be most optimally "extracted" from a system of $N$ identically prepared spin- $1 / 2$ particles. The ability of the system to retain information about the phase (time) depends very much on the choice of the initial reference state $\hat{\Omega}$. For instance, if this state is an eigenstate of the total Hamiltonian, the system is not able to record (keep) time information. In Ref. [2] we addressed the question of which is the most appropriate initial state $\hat{\Omega}$ of the $N$ spin- $1 / 2$ particle which "keeps" the record of the phase in the most reliable way. In other words, what are the optimal quantum clocks, and what is the performance of such quantum clocks when compared with classical clocks.

In the present paper we investigate another aspect of this comparison. That is, we discuss the "robustness" of quantum clocks with respect to repeated measurements performed on them. Classical clocks, as all classical objects, do not change their state or behavior when they are observed. As we stated above, this is no longer true for quantum objects. This has consequences for the functioning of our proposed quantum clocks. In particular, one can ask whether quantum clocks may be robust enough in the sense that repeated readout of the time, let us say by many independent and noncommunicating observers, can provide reliable information (if any) about the time to all of them. In order to find quantitative answers to our questions, let us recall briefly the details of how time is read out from our quantum clocks.

In general, a quantum-mechanical measurement is described by a positive operator value measure (POVM) $[1,5,6]$ which is a set $\left\{\hat{O}_{r}\right\}_{r=1}^{R}$ of positive Hermitian operators, such that $\Sigma_{r} \hat{O}_{r}=\hat{1}$. Because such a measurement is in general nondeterministic, with each outcome $r$ of the measurement we associate an estimate $t_{r}$ of the time elapsed. The differ- ence between the estimated time $t_{r}$ and the true time $t$ is quantified by a cost function $f\left(t_{r}-t\right)$ [6]. Here we note that because of the periodicity of the clock, $f$ has to be periodic. We also take $f(t)$ to be an even function to ensure a nonzero average. Our task is to minimize the mean value of the cost function

$$
\bar{f}=\sum_{r} \int_{0}^{2 \pi} p_{1}(r \mid t) f\left(t_{r}-t\right) \frac{d t}{2 \pi},
$$

where

$$
p_{1}(r \mid t)=\operatorname{Tr}\left[\hat{O}_{r} \hat{\Omega}(t)\right] .
$$

Following the discussion in Ref. [2], we chose the cost function to be $4 \sin ^{2} t / 2$, which for small values of the mean cost can be approximated as $\bar{f} \simeq \Delta t^{2}$.

Holevo [6] originally considered covariant measurements in which times $t_{r}$ take a continuum of values between 0 and $2 \pi$. But, as shown in Ref. [4], the completeness relation can also be satisfied by taking a discrete set of times $t_{r}$ $=2 \pi r /(N+1), r=0, \ldots, N$. In this case the Hermitian operators $\hat{O}_{r}$ can be taken in the form

$$
\hat{O}_{r}=\left|\Psi_{r}\right\rangle\left\langle\Psi_{r}\right|,
$$

such that $\Sigma_{r} \hat{O}_{r}=\hat{1}$, where

$$
\left|\Psi_{r}\right\rangle=e^{i t_{r} \hat{H}}\left|\Psi_{0}\right\rangle, \quad\left|\Psi_{0}\right\rangle=\frac{1}{\sqrt{N+1}} \sum_{m=0}^{N}|m\rangle,
$$

which can be rewritten as

$$
\left|\Psi_{r}\right\rangle=\frac{1}{\sqrt{N+1}} \sum_{m=0}^{N} e^{i[2 \pi /(N+1)] r m}|m\rangle .
$$

The phase states $\left|\Psi_{r}\right\rangle$ [7] form an orthonormal basis of the Hilbert space, and the corresponding measurement is therefore a von Neumann measurement. This is important for applications, because it means that it is not necessary to use an ancilla to make the optimal measurement. Moreover, as follows from the von Neumann projection postulate, the state immediately after the measurement is uniquely determined.

Once we have specified the optimal measurement we have to specify the initial (reference) state $\hat{\Omega}$ of our system. As discussed in Ref. [2], by an appropriate choice of this state one can substantially improve the performance of quantum clocks. However, this concerns the estimation performed by the first observer (see below). The subsequent observers will actually always observe only rotated phase states. These are generated in the von Neumann measurement performed by the previous observer and subsequent time evolution. Therefore, in order to simplify our calculations we will assume that the initial (reference) state $\hat{\Omega}$ is the phase state $\hat{\Omega}$ $=\left|\Psi_{0}\right\rangle\left\langle\Psi_{0}\right|$ given by Eq. (7). This initial state will evolve in time as $\hat{\Omega}(t)=\left|\Psi_{0}(t)\right\rangle\left\langle\Psi_{0}(t)\right|$, where 


$$
\left|\Psi_{0}(t)\right\rangle=\frac{1}{\sqrt{N+1}} \sum_{m=0}^{N} e^{-i t m}|m\rangle .
$$

Let us study now how independent observers measured a system of $N$ spin-1/2 particles initially prepared in an unknown state obtained by the rotation of the reference state [Eq. (7)]. As far as the first observer was concerned, the problem was already solved (see Ref. [4]) and the mean cost [Eq. (4)] could be calculated. Taking into account that the optimal phase (time) measurement is realized via the projectors $\hat{O}_{r}=\left|\Psi_{r}\right\rangle\left\langle\Psi_{r}\right|$ with the phase states $\left|\Psi_{r}\right\rangle$ given by Eq. (8), we can express the mean cost $\bar{f}_{1}$ after the first measurement (therefore we use the subscript 1 ) as

$$
\bar{f}_{1}=4 \sum_{r=0}^{N} \int_{0}^{2 \pi} \frac{d t}{2 \pi}\left|\sum_{m=0}^{N} e^{i\left(t_{r}-t\right) m}\right|^{2} \sin ^{2} \frac{\left(t_{r}-t\right)}{2} .
$$

After the integration is performed, we find that the mean cost as a function of number $N$ of spin-1/2 particles is given by the expression

$$
\Delta t^{2} \simeq \bar{f}_{1}(N)=2\left[1-\frac{N}{N+1}\right]=\frac{2}{N+1} .
$$

We see that the mean cost when a single measurement is performed $(N=1)$ takes the value $\bar{f}_{1}(N=1)=1$. Conversely, for $N \rightarrow \infty$ the mean cost is equal to zero. Specifically, for large $N$ the variance $\Delta t$ goes to zero as $1 / \sqrt{N}$. This is far from being optimal. As shown in Ref. [2], in order to make this variance minimal we should take the reference state to be

$$
\left|\Psi_{o p t}\right\rangle \simeq \frac{\sqrt{2}}{\sqrt{N+1}} \sum_{m=0}^{N} \sin \frac{\pi(m+1 / 2)}{N+1}|m\rangle .
$$

In this case the cost decreases for large $N$ as $\bar{f}_{\text {opt }} \simeq \pi^{2} /(N$ $+1)^{2}$ corresponding to $\Delta t_{\text {opt }} \simeq \pi /(N+1)$. Nevertheless, as our task is to study how much information subsequent observers can gain we are not over-worried about the optimality of the preparation of the reference state. Our further result can be understood as a lower bound, and the optimization can be performed rather straightforwardly in any case.

Now we turn our attention to subsequent observers. We have assumed that our observers do not communicate. If they do then the first observer can broadcast the result of his measurement (or, what is equivalent, he can broadcast the orientation of his apparatus) and there is no need for subsequent observers to perform any measurement, because they know that they cannot perform better than this first observer. To describe the mean cost of the estimation of subsequent observers, in Eq. (4) we have to modify the conditional probability distribution $p_{1}(r \mid t)=\operatorname{Tr}\left[\hat{O}_{r} \hat{\Omega}(t)\right]$ characterizing the measurement statistics of the observer. This is because the $(k+1)$ st observer does not observe the original state $\hat{\Omega}(t)$. He can only measure the state generated via the measurement performed by the previous $k$ th observer. Taking into account the projective character of the measurement, this state is one of the states $\left|\Psi_{r}\right\rangle$. In addition, the following random factors enter the game: First, the $(k+1)$ st observer does not have complete information about the choice of the measuring apparatus of the $k$ th observer. Although all observers possess an optimal measuring apparatus of the same construction (corresponding to the optimal von Neumann measurement) there is one parameter they can choose at random. That is, if we take the POVM characterized by the set of projectors $\hat{O}_{r}=\left|\Psi_{r}\right\rangle\left\langle\Psi_{r}\right|, r=0, \ldots, N$, and rotate them all by the same transformation $\hat{U}(\alpha)=\exp \{-i \alpha \hat{H}\}$, we obtain a new POVM, $\hat{O}_{r}^{\alpha}=\hat{U}(\alpha) \hat{O}_{r} \hat{U}^{\dagger}(\alpha)$, which also corresponds to the optimal measuring apparatus. It is this information about the angle $\alpha^{\prime} \in\langle 0,2 \pi\rangle$ characterizing the " "actual orientation" of the $k$ th measuring apparatus which is not available to the $(k+1)$ st observer. The second piece of information which is not available to the $(k+1)$ st observer is the knowledge of which of the possible outcomes $r^{\prime}$ of the measurement was detected by the $k$ th observer. Finally, the actual time $t^{\prime}$ when this measurement was performed is also unknown (however, as we will soon see, this is not important for our consideration). Taking these random factors into account, the required conditional probability distribution $p_{k+1}(r \mid t, \alpha)$ (we have included the parameter $\alpha$ in the conditional probability distribution) reads

$$
\begin{aligned}
p_{k+1}(r \mid t, \alpha)= & \sum_{r^{\prime}=0}^{N} \int_{0}^{2 \pi} p_{k}\left(r^{\prime} \mid t^{\prime}, \alpha^{\prime}\right) \frac{d \alpha^{\prime}}{2 \pi} \\
& \times \operatorname{Tr}\left[\hat{O}_{r}^{\alpha} \hat{U}\left(t-t^{\prime}\right) \hat{O}_{r^{\prime}}^{\alpha^{\prime}} \hat{U}^{\dagger}\left(t-t^{\prime}\right)\right] .
\end{aligned}
$$

It is easily seen that this can be simplified as

$$
p_{k+1}(r \mid t, \alpha)=\operatorname{Tr}\left[\hat{\Omega}_{k+1}(t) \hat{O}_{r}^{\alpha}\right],
$$

where

$$
\hat{\Omega}_{k+1}(t)=\sum_{r^{\prime}=0}^{N} \int_{0}^{2 \pi} p_{k}\left(r^{\prime} \mid t, \alpha^{\prime}\right) \hat{O}_{r^{\prime}}^{\alpha^{\prime}} \frac{d \alpha^{\prime}}{2 \pi}
$$

The last transformation is possible because $p_{k}\left(r^{\prime} \mid t^{\prime}, \alpha^{\prime}\right)$ $=p_{k}\left(r^{\prime} \mid t, \alpha^{\prime}+t-t^{\prime}\right)$ and the integration with respect to $\alpha^{\prime}$ ensures that the shift $\left(t-t^{\prime}\right)$ is irrelevant.

Now we define the mean cost of the $(k+1)$ st measurement as

$$
\bar{f}_{k+1}(N)=\sum_{r} \int_{0}^{2 \pi} p_{k+1}(r \mid t, \alpha) f\left(t_{r}-t\right) \frac{d t}{2 \pi} .
$$

Obviously the choice of $\alpha$ (the orientation of the apparatus) does not affect the mean cost of the measurement under given circumstances. On the other hand, it definitely affects the state into which system collapses after the projective measurement $\hat{O}_{r}^{\alpha}$.

Before we present a general solution for the mean cost [Eq. (16)], we analyze a simple example: Let us assume just a single qubit, i.e., $N=1$. This qubit is first prepared in the state $|0\rangle$, and then after the application of the Hadamard 
transform it is "rotated" into a superposition state $|\psi\rangle$ $=(|0\rangle+|1\rangle) / \sqrt{2}$, which evolves according to the free Hamiltonian as

$$
|\psi(t)\rangle=\left(|0\rangle+e^{-i t}|1\rangle\right) / \sqrt{2}
$$

i.e., $\hat{\Omega}_{1}(t)=|\psi(t)\rangle\langle\psi(t)|$. The two projectors which describe the measurement apparatus used by the first observer are defined above, i.e., $\hat{O}_{r}=\left|\Psi_{r}\right\rangle\left\langle\Psi_{r}\right|$, where in the case of a single qubit

$$
\left|\Psi_{0}\right\rangle=\frac{1}{\sqrt{2}}(|0\rangle+|1\rangle), \quad\left|\Psi_{1}\right\rangle=\frac{1}{\sqrt{2}}(|0\rangle-|1\rangle) .
$$

In this case, obviously, $t_{0}=0$ and $t_{1}=\pi$. The probability that the system $|\psi(t)\rangle$ is measured in the state $\left|\Psi_{r}\right\rangle$ is

$$
p_{1}(r \mid t)=\left|\left\langle\Psi_{r} \mid \psi(t)\right\rangle\right|^{2}=\frac{1}{2}(1 \pm \cos t), \quad r=0,1 .
$$

Thus the mean cost of the first measurement is

$$
\begin{aligned}
\bar{f}_{1} & =2 \int_{0}^{2 \pi}\left[(1+\cos t) \sin ^{2} \frac{t}{2}+(1-\cos t) \cos ^{2} \frac{t}{2}\right] \frac{d t}{2 \pi} \\
& =2\left(1-\frac{1}{2}\right)
\end{aligned}
$$

which is equal to 1 , and is in accordance with the general result given by Eq. (11). The system after the measurement is either in the state $\left|\Psi_{0}\right\rangle$ or in the state $\left|\Psi_{1}\right\rangle$.

Now assume that the second observer is going to perform a measurement under the conditions described above. Since he does not know the result of the previous measurement, and does not know the orientation of the first apparatus, he has to assume that the state he is going to measure has the form given by Eq. (15), i.e.,

$$
\hat{\Omega}_{2}(t)=\sum_{r^{\prime}=0}^{1} \int_{0}^{2 \pi} p_{1}\left(r^{\prime} \mid t, \alpha^{\prime}\right) \hat{O}_{r^{\prime}}^{\alpha^{\prime}} \frac{d \alpha^{\prime}}{2 \pi} .
$$

This means he has to average over all possible orientations of the first apparatus as well as over all possible outcomes of the first measurement. We first specify the projectors $\hat{O}_{r^{\prime}}^{\alpha^{\prime}}=\left|\Psi_{r^{\prime}}\left(\alpha^{\prime}\right)\right\rangle\left\langle\Psi_{r^{\prime}}\left(\alpha^{\prime}\right)\right|$, where $\left|\Psi_{r^{\prime}}\left(\alpha^{\prime}\right)\right\rangle$ $=\exp \left(-i \alpha^{\prime} \hat{H}\right)\left|\Psi_{r^{\prime}}\right\rangle$. These states in the case of a singe qubit read

$$
\begin{aligned}
& \left|\Psi_{0}\right\rangle=\frac{1}{\sqrt{2}}\left(|0\rangle+e^{-i \alpha^{\prime}}|1\rangle\right), \\
& \left|\Psi_{1}\right\rangle=\frac{1}{\sqrt{2}}\left(|0\rangle-e^{-i \alpha^{\prime}}|1\rangle\right) .
\end{aligned}
$$

The corresponding probabilities $p_{1}\left(r^{\prime} \mid t, \alpha^{\prime}\right)$ then read

$$
p_{1}\left(r^{\prime} \mid t, \alpha^{\prime}\right)=\left|\left\langle\Psi_{r} \mid \psi(t)\right\rangle\right|^{2}=\frac{1}{2}\left[1 \pm \cos \left(t-\alpha^{\prime}\right)\right],
$$

where $+(-)$ stands for $r^{\prime}=0(1)$. Consequently, using Eq. (15), we find

$$
\hat{\Omega}_{2}(t)=\frac{1}{4} \hat{I}+\frac{1}{2} \hat{\Omega}_{1}(t) .
$$

When we insert this expression into Eq. (14) we find the probabilities $p_{2}(r \mid t, \alpha)$ (for definiteness, in what follows we chose $\alpha=0)$ :

$$
p_{2}(r \mid t, \alpha)=\frac{1}{2}\left(1 \pm \frac{1}{2} \cos t\right), \quad r=0,1 .
$$

Once this is done we utilize Eq. (16), and for the mean cost of the second measurement we find the expression

$$
\begin{aligned}
\bar{f}_{2} & =\sum_{r=0}^{1} \int_{0}^{2 \pi} p_{2}(r \mid t, \alpha) f\left(t_{r}-t\right) \frac{d t}{2 \pi} \\
& =2 \int_{0}^{2 \pi[}\left[\left(1+\frac{\cos t}{2}\right) \sin ^{2} \frac{t}{2}+\left(1-\frac{\cos t}{2}\right) \cos ^{2} \frac{t}{2}\right] \frac{d t}{2 \pi} \\
& =2\left[1-\left(\frac{1}{2}\right)^{2}\right]=\frac{3}{2}
\end{aligned}
$$

As expected, the mean cost of the second observation is larger than for the first one, but is still smaller than the cost associated with a random guess $(\bar{f}=2)$. This means that the second observer can gain nontrivial information about the original preparation of the qubit.

Using the iterative definition given by Eqs. (14) and (15), together with the definition for the mean cost [Eq. (4)], we calculate the precision of the measurement of time performed with the quantum clocks as a function of the number of qubits $N$ and the number of subsequent observers $k$ :

$$
\bar{f}_{k}(N)=2\left[1-\left(\frac{N}{N+1}\right)^{k}\right]
$$

This is the main result of our paper. We stress that the above result holds for the reference state corresponding to the phase state [Eq. (7)], and the case in which observers have no $a$ priori knowledge about the initial-state preparation. This can be generalized to the case when the initial reference state is taken to be the optimal state [Eq. (12)]—unfortunately, in this case we are not able to find a solution in an elegant closed analytical form.

Let us summarize our results. We have shown that by performing a measurement on quantum clocks which were already measured independently, observers can still obtain nontrivial information about the original preparation of the quantum system. The larger the ensemble $(N)$, the more robust the quantum system with respect to subsequent measurements. Obviously, as follows from Eq. (27) for the $(k$ $+1)$ st observer, the mean cost of the estimation will be 
larger than for the $k$ th observer. From the point of view of information stored in the system, in the large- $N$ limit the quantum system behaves very classically, i.e., an infinite number of independent observers who have no prior knowledge about the state preparation can precisely measure the state of the system.
One of us (V.B.) thanks Rado Derka and Serge Massar for helpful discussions. This work was supported in part by the Royal Society, the IST Projects EQUIP (IST-1999-11053) and QUBITS (IST-1999-13021), the U.K. Engineering and Physical Sciences Research Council, and the CREST, Research Team for Interacting Career Electronics.
[1] A. Peres, Quantum Theory: Concepts and Methods (Kluwer, Dordrecht, 1993).

[2] V. Bužek, R. Derka, and S. Massar, Phys. Rev. Lett. 82, 2207 (1999). Here we do not address questions concerning the improvement of frequency standards obtained through the use of entanglement, as discussed by S. F. Huelga et al., ibid. 79, 3865 (1997).

[3] D. J. Wineland, C. Monroe, W. M. Itano, D. Leibfried, B. E. King, and D. M. Meekhof, J. Res. Natl. Inst. Stand. Technol. 103, 259 (1998).
[4] R. Derka, V. Bužek, and A. K. Ekert, Phys. Rev. Lett. 80, 1571 (1998).

[5] C. W. Helstrom, Quantum Detection and Estimation Theory (Academic Press, New York, 1976).

[6] A. S. Holevo, Probabilistic and Statistical Aspects of Quantum Theory (North-Holland, Amsterdam, 1982).

[7] These states are eigenstates of the Hermitian phase operator as introduced by D. T. Pegg and S. M. Barnett, Europhys. Lett. 6, 483 (1988). 